\title{
Naturally light Dirac neutrinos from SU(6)
}

\author{
Ernest Ma \\ Department of Physics and Astronomy, University of California, \\ Riverside, California 92521, U.S.A. \\ E-mail: ma@phyun8.ucr.edu
}

ABSTRaCT: A known mechanism for obtaining naturally light Dirac neutrinos is implemented in the context of $\mathrm{SU}(6) \rightarrow \mathrm{SU}(5) \times \mathrm{U}(1)_{N}$.

Keywords: GUT, Neutrino Physics

ArXiv EPRINT: 2103.02072 


\section{Contents}

1 Introduction $\quad 1$

2 Description of model 2

3 Residual symmetries $\quad 3$

4 Model characteristics $\quad 4$

5 Concluding remarks $\quad 4$

\section{Introduction}

Whether or not neutrinos are Majorana or Dirac is a fundamental issue in particle physics. Experimentally, there is still no evidence for one or the other, although it is known that at least two neutrinos must have masses, because of neutrino oscillations [1]. Theoretically, the standard model (SM) requires only neutrinos in the left-handed $\mathrm{SU}(2)_{L} \times \mathrm{U}(1)_{Y}$ doublets $(\nu, l)_{L}$. The singlet $\nu_{R}$ is not necessary because it is trivial under the SM gauge group $\mathrm{SU}(3)_{C} \times \mathrm{SU}(2)_{L} \times \mathrm{U}(1)_{Y}$. To have a Dirac neutrino, $\nu_{R}$ must exist. To justify its existence, a gauge extension of the $\mathrm{SM}$ is often considered, either $\mathrm{U}(1)_{Y} \rightarrow \mathrm{U}(1)_{Y} \times \mathrm{U}(1)_{B-L}$ [2] or $\mathrm{U}(1)_{Y} \rightarrow \mathrm{SU}(2)_{R} \times \mathrm{U}(1)_{B-L}[3]$, which may be incorporated into the grand unified structure of $\mathrm{SO}(10)$. The breaking of $\mathrm{U}(1)_{B-L}$ [and $\mathrm{SU}(2)_{R}$ ] is usually assumed without hesitation to allow $\nu_{R}$ to obtain a large Majorana mass, so that $\nu_{L}$ gets a tiny seesaw Majorana mass, as is well-known.

There is actually another option. This breaking does not have to be $\Delta L=2$. If it is $\Delta L=3$ for example, then neutrinos are Dirac. This was first pointed out [4] for a general $\mathrm{U}(1)_{X}$ symmetry and applied [5] to $\mathrm{U}(1)_{L}$ for Dirac neutrinos. However, this mechanism does not by itself explain why the neutrino Higgs Yukawa couplings are so small.

To overcome this problem, the mechanism of ref. [6] is the simplest solution. The idea is to have at least two Higgs doublets, say $\Phi=\left(\phi^{+}, \phi^{0}\right)$ and $\eta=\left(\eta^{+}, \eta^{0}\right)$ which are distinguished by some symmetry, so that $\bar{\nu}_{R} \nu_{L}$ couples to $\eta^{0}$, but not $\phi^{0}$. This symmetry is then broken by the $\mu^{2} \Phi^{\dagger} \eta$ term, under the condition that $m_{\Phi}^{2}<0$ and $m_{\eta}^{2}>0$ and large. In that case, the vacuum expectation value $\left\langle\eta^{0}\right\rangle$ is given by $-\mu^{2}\left\langle\phi^{0}\right\rangle / m_{\eta}^{2}$, which is naturally small, implying thus a very small Dirac neutrino mass. In the original application [6], $\nu_{R}$ is also allowed a large Majorana mass, hence the mass of $\nu_{L}$ is doubly suppressed. In that case, $m_{\eta}$ could well be of order $1 \mathrm{TeV}$. On the other hand, if the symmetry and the particle content are such that $\nu_{R}$ is prevented from having a Majorana mass, then a much larger $m_{\eta}$ works just as well for a tiny Dirac neutrino mass.

Recently this idea has been applied $[7,8]$ using a gauge $\mathrm{U}(1)_{D}$ symmetry under which the SM particles do not transform, but $\nu_{R}$ and other fermion singlets do. The $\mathrm{U}(1)_{D}$ 


\begin{tabular}{|c|c|c|c|c|c|c|}
\hline fermion & $\mathrm{SU}(5)$ & $\mathrm{SU}(3)_{C}$ & $\mathrm{SU}(2)_{L}$ & $\mathrm{U}(1)_{Y}$ & $\mathrm{U}(1)_{N}$ & $\mathrm{U}(1)_{X}$ \\
\hline$d^{c}$ & $5^{*}$ & $3^{*}$ & 1 & $1 / 3$ & -1 & 1 \\
$(\nu, e)$ & $5^{*}$ & 1 & 2 & $-1 / 2$ & -1 & 1 \\
$N$ & 1 & 1 & 1 & 0 & 5 & 1 \\
\hline$D^{c}$ & $5^{*}$ & $3^{*}$ & 1 & $1 / 3$ & -1 & 2 \\
$\left(E^{0}, E^{-}\right)$ & $5^{*}$ & 1 & 2 & $-1 / 2$ & -1 & 2 \\
$\nu^{c}$ & 1 & 1 & 1 & 0 & 5 & 2 \\
\hline$(u, d)$ & 10 & 3 & 2 & $1 / 6$ & 2 & 0 \\
$u^{c}$ & 10 & $3^{*}$ & 1 & $-2 / 3$ & 2 & 0 \\
$e^{c}$ & 10 & 1 & 1 & 1 & 2 & 0 \\
\hline$D$ & 5 & 3 & 1 & $-1 / 3$ & -4 & 0 \\
$\left(E^{+}, \bar{E}^{0}\right)$ & 5 & 1 & 2 & $1 / 2$ & -4 & 0 \\
\hline
\end{tabular}

Table 1. Fermion content of $\mathrm{SU}(6) \rightarrow \mathrm{SU}(5) \times \mathrm{U}(1)_{N}$ model.

symmetry is broken by singlet scalars which transform only under $\mathrm{U}(1)_{D}$. The bridge between the SM and this new sector is a set of Higgs doublets which transform under both. The particle content is chosen such that global lepton number is conserved as well as a dark parity or dark number.

In this paper, instead of the ad hoc $\mathrm{U}(1)_{D}$ symmetry, $\nu_{R}$ is identified as part of the fundamental representation of $\mathrm{SU}(6)$ which breaks to $\mathrm{SU}(5) \times \mathrm{U}(1)_{N}$. Following a recent analysis [9], it is shown how naturally small Dirac neutrino masses occur in this context.

\section{Description of model}

Starting with the well-known SU(5) model [10] of grand unification, an extension to SU(6) is straightforward $[11,12]$. Instead of having the anomaly-free combination of $5^{*}$ and 10 under $\mathrm{SU}(5)$ for each family, there should be now two $6^{*}=\left(5^{*},-1\right)+(1,5)$ and one $15=(10,2)+(5,-4)$ under $\mathrm{SU}(6) \rightarrow \mathrm{SU}(5) \times \mathrm{U}(1)_{N}$. Let

$$
6_{F 1}^{*}=\left(\begin{array}{c}
d^{c} \\
d^{c} \\
d^{c} \\
e \\
\nu \\
N
\end{array}\right), \quad 6_{F 2}^{*}=\left(\begin{array}{c}
D^{c} \\
D^{c} \\
D^{c} \\
E^{-} \\
E^{0} \\
\nu^{c}
\end{array}\right), \quad 15_{F}=\left(\begin{array}{cccccc}
0 & u^{c} & u^{c} & -u & -d & -D \\
-u^{c} & 0 & u^{c} & -u & -d & -D \\
u^{c} & -u^{c} & 0 & -u & -d & -D \\
u & u & u & 0 & -e^{c} & -E^{+} \\
d & d & d & e^{c} & 0 & -\bar{E}^{0} \\
D & D & D & E^{+} & \bar{E}^{0} & 0
\end{array}\right) .
$$

Their $\mathrm{SU}(3)_{C} \times \mathrm{SU}(2)_{L} \times \mathrm{U}(1)_{Y} \times \mathrm{U}(1)_{N}$ assignments are listed in table 1. Note that all are left-handed and $\nu^{c}$ sits in a different multiplet from $\nu$, whereas both are in the same multiplet in the recent proposal of ref. [13]. The added $\mathrm{U}(1)_{X}$ is a global symmetry imposed on the dimension-four couplings of the resulting Lagrangian, but softly broken by 


\begin{tabular}{|c|c|c|c|c|c|c|c|}
\hline$\langle$ scalar $\rangle$ & $\mathrm{SU}(6)$ & $\mathrm{SU}(5)$ & $\mathrm{SU}(3)_{C}$ & $\mathrm{SU}(2)_{L}$ & $\mathrm{U}(1)_{Y}$ & $\mathrm{U}(1)_{N}$ & $\mathrm{U}(1)_{X}$ \\
\hline$u_{1}$ & 35 & 24 & 1 & 1 & 0 & 0 & 0 \\
$u_{2}$ & 6 & 1 & 1 & 1 & 0 & -5 & 2 \\
$u_{3}$ & $21^{*}$ & 1 & 1 & 1 & 0 & 10 & 2 \\
\hline$v_{1}$ & 84 & 5 & 1 & 2 & $1 / 2$ & 1 & 1 \\
$v_{2}$ & $15^{*}$ & $5^{*}$ & 1 & 2 & $-1 / 2$ & 4 & 0 \\
$v_{3}$ & $15^{*}$ & $5^{*}$ & 1 & 2 & $-1 / 2$ & 4 & 3 \\
\hline
\end{tabular}

Table 2. Scalar content of $\mathrm{SU}(6) \rightarrow \mathrm{SU}(5) \times \mathrm{U}(1)_{N}$ model.

bilinear and trilinear scalar terms. The scalars which break the SU(6) symmetry and allow these fermions to acquire masses are listed in table 2 . The $35_{S}$ breaks $\mathrm{SU}(6)$ at the garnd unification scale $u_{1}$ to $\mathrm{SU}(3)_{C} \times \mathrm{SU}(2)_{L} \times \mathrm{U}(1)_{Y} \times \mathrm{U}(1)_{N}$. The $6_{S}$ breaks $\mathrm{U}(1)_{N}$ at a lower scale. Because it is charged under $\mathrm{U}(1)_{X}$, its only allowed coupling is $6_{S}^{*} \times 6_{F_{2}}^{*} \times 15_{F}$. Hence $D^{c} D$ and $E^{-} E^{+}+E^{0} \bar{E}^{0}$ masses are proportional to $u_{2}$. The $21_{S}^{*}$ also breaks $\mathrm{U}(1)_{N}$. The $21_{S} \times 6_{F 1}^{*} \times 6_{F 1}^{*}$ term yields Majorana masses $N N$ proportional to $u_{3}$. The electroweak $\mathrm{SU}(2)_{L} \times \mathrm{U}(1)_{Y}$ symmetry is broken by three Higgs doublets. The $84_{S}^{*} \times 6_{F 1}^{*} \times 15_{F}$ term yields masses for $d^{c} d, e e^{c}, N \bar{E}^{0}$ which are proportional to $v_{1}$. The $15_{S} \times 15_{F} \times 15_{F}$ term yields masses for $u^{c} u$ proportional to $v_{2}$, whereas the $15_{S} \times 6_{F 1}^{*} \times 6_{F 2}^{*}$ term yields masses for $\nu \nu^{c}, N E^{0}$ proportional to $v_{3}$. Let these three Higgs doublets be named $\Phi_{1,2}$ and $\eta$ respectively. To obtain a small $v_{3}$, it is clear that $m_{\eta}^{2}$ must be positive and large, as discussed in the introduction. Now both $\eta$ and $\Phi_{2}$ come from $15_{S}$ but are distinguished by $\mathrm{U}(1)_{X}$. Hence the soft term $\mu^{2} \Phi_{2}^{\dagger} \eta$ breaking $\mathrm{U}(1)_{X}$ by 3 units is available to make the Dirac neutrino masses very small. Note that $\nu^{c}$ does not acquire a Majorana mass because of the absence of a scalar $21^{*}$ with 4 units of charge under $\mathrm{U}(1)_{X}$. The two soft trilinear terms $84_{S} \times 6_{S} \times 15_{S}^{*}$ break $\mathrm{U}(1)_{X}$ by 3 and 6 units, whereas $6_{S} \times 6_{S} \times 21_{S}^{*}$ does it by 6 units.

\section{Residual symmetries}

Because of the $\mathrm{U}(1)_{X}$ symmetry, Yukawa terms are restricted so that residual symmetries exist for the fermions of this model at the level of $\mathrm{SU}(3)_{C} \times \mathrm{SU}(2)_{L} \times \mathrm{U}(1)_{Y} \times \mathrm{U}(1)_{N}$. The usual baryon number $B$ and lepton number $L$ are then conserved, together with a dark parity $Z_{2}^{D}$ which may be identified as $(-1)^{3 B+L+2 j}$, where $j$ is the intrinsic spin of the particle, as shown in table 3 .

The $3 \times 3$ mass matrix spanning $\left(N, E^{0}, \bar{E}^{0}\right)$ is of the form

$$
\mathcal{M}_{N E}=\left(\begin{array}{ccc}
f_{N} u_{3} & f_{N E} v_{3} & f_{N E}^{\prime} v_{1} \\
f_{N E} v_{3} & 0 & f_{E} u_{2} \\
f_{N E}^{\prime} v_{1} & f_{E} u_{2} & 0
\end{array}\right) .
$$

The lightest mass eigenstate is possible dark matter. However as shown in ref. [9], because of its $\mathrm{SU}(6)$ antecedent, $N$ may decay through a superheavy gauge boson in analogy to proton decay. Note also that $v_{3}$ is very small. If $u_{3}$ is absent, then $N$ gets a small seesaw 


\begin{tabular}{|c|c|c|c|}
\hline fermion & $B$ & $L$ & $Z_{2}^{D}$ \\
\hline$u, d$ & $1 / 3$ & 0 & + \\
$\nu, l$ & 0 & 1 & + \\
\hline$D$ & $1 / 3$ & 1 & - \\
$N, E^{0}, E^{-}$ & 0 & 0 & - \\
\hline
\end{tabular}

Table 3. Residual Symmetries of $\mathrm{SU}(6) \rightarrow \mathrm{SU}(5) \times \mathrm{U}(1)_{N}$ model.

mass proportional to $v_{1} v_{3} / u_{2}$. This makes it a candidate for very light freeze-in dark matter, as described in ref. [8]. In such a scenario, the $21^{*}$ scalar is not required.

In the $15_{S} \times 6_{F 1}^{*} \times 6_{F 2}^{*}$ coupling, the $(5,-4)$ component of $15_{S}$ contains $\eta^{0}$ which couples to $\nu \nu^{c}$ as well as $N E^{0}$. It also contains the scalar color triplet $\zeta=(3,1,-1 / 3,-4)$ which couples to both $d^{c} \nu^{c}$ and $N D^{c}$. Hence $D^{c}$ must have $L=-1$ and $N$ must have $L=0$. Note that $\zeta$ has then $B=1 / 3$ and $L=1$, so that its dark parity, i.e. $(-1)^{3 B+L+2 j}$, is even as expected. The dark quark $D$ decays through $\zeta$ to $d \nu N$. This process is a three-body decay with two invisible particles and not easy to observe.

\section{Model characteristics}

Below the breaking scale $u_{2,3}$ of $\mathrm{U}(1)_{N}$, the particle content of the proposed model is that of the SM with the following changes. The neutrinos are Dirac particles. There are two Higgs doublets, one coupling to $\bar{u} u$ and the other to $\bar{d} d$ and $\bar{e} e$. A third Higgs doublet is very heavy and not observable, but it has a tiny vacuum expectation value and couples to $\bar{\nu} \nu$. There may also be a very light neutral Majorana fermion $N$ which is freeze-in dark matter in the case that $u_{3}=0$.

The above particles all interact with a new heavy gauge boson $Z_{N}$ coming from $\mathrm{U}(1)_{N}$, according to their charges given in tables 1 and 2 . The present collider limit [1] of $Z_{N}$ is estimated to be a few $\mathrm{TeV}$. At or above the $Z_{N}$ mass scale, particles of the dark sector as well as one or more Higgs singlets should appear. The $D$ quark may be easily produced at the collider and decay to the $d$ quark plus a neutrino and the dark $N$. The doublet $\left(E^{0}, E^{-}\right)$dark fermions also decay to $N$ plus a scalar or the $W^{-}$gauge boson. More details are in ref. [9].

\section{Concluding remarks}

It has been shown that a framework exists for naturally small Dirac neutrino masses in the context of $\mathrm{SU}(6) \rightarrow \mathrm{SU}(5) \times \mathrm{U}(1)_{N}$, where the right-handed neutrino singlet $\nu^{c}$ is embedded as shown in eq. (1). With the implementation of a softly broken global $\mathrm{U}(1)_{X}$ symmetry as given in tables 1 and 2, the residual symmetries of baryon number $B$ and lepton number $L$ are preserved as given in table 3 , with dark parity identified as $Z_{2}^{D}=(-1)^{3 B+L+2 j}$. The reason for a conserved lepton number parallels that of ref. [4], i.e. the interplay of $\mathrm{U}(1)_{X}$ and the chosen $\mathrm{SU}(6)$ representations makes it impossible for $\nu^{c}$ to be a Majorana fermion. 
The dark sector consists of $D$ leptoquarks and $L=0$ fermions $N$ and the vectorlike doublet $\left(E^{0}, E^{-}\right)$. Whereas $N$ mixes with $E^{0}$ and $\bar{E}^{0}$, the former may be almost a mass eigensate and considered as dark matter. It is presumably of order a few $\mathrm{TeV}$, but if the $21^{*}$ scalar is removed from table 2 , then $u_{3}=0$ and eq. (2) yields a very small mass for $N$ which then becomes freeze-in dark matter.

Suppose the $\mathrm{U}(1)_{N}$ breaking scale is much higher than a few $\mathrm{TeV}$, then only the SM particles are observable, with the important difference that neutrinos are Dirac, and there are two Higgs doublets. The other possible addition is the Majorana fermion $N$ as freeze-in dark matter. If $u_{3}=0$, then its mass is proportional to $v_{1} v_{3} / u_{2}$ as remarked earlier, which is perhaps too small because $v_{3}$ is responsible for the Dirac neutrino mass and $u_{2}$ is now assumed to be very large. However, the $21^{*}$ scalar may be retained, and $u_{3} \neq 0$ rendered small (but not too small) by the same mechanism [6] which makes $v_{3}$ small, i.e. of the form $-\mu_{23} u_{2}^{2} / m_{3}^{2}$ from the term $6_{S} \times 6_{S} \times 21_{S}^{*}$, where $m_{3}$ is the mass of the scalar $21^{*}$.

\section{Acknowledgments}

This work was supported in part by the U. S. Department of Energy Grant No. DESC0008541.

Open Access. This article is distributed under the terms of the Creative Commons Attribution License (CC-BY 4.0), which permits any use, distribution and reproduction in any medium, provided the original author(s) and source are credited.

\section{References}

[1] Particle Data Group collaboration, Review of Particle Physics, PTEP 2020 (2020) 083C01 [INSPIRE].

[2] A. Davidson, $B-L$ as the fourth color within an $\mathrm{SU}(2)_{L} \times \mathrm{U}(1)_{R} \times \mathrm{U}(1)$ model, Phys. Rev. $D 20$ (1979) 776.

[3] R. Marshak and R. Mohapatra, Quark-lepton symmetry and B - L as the $\mathrm{U}(1)$ generator of the electroweak symmetry group, Phys. Lett. B 91 (1980) 222.

[4] E. Ma, I. Picek and B. Radovčić, New Scotogenic Model of Neutrino Mass with U(1) ${ }_{D}$ Gauge Interaction, Phys. Lett. B $\mathbf{7 2 6}$ (2013) 744 [arXiv:1308.5313] [INSPIRE].

[5] E. Ma and R. Srivastava, Dirac or inverse seesaw neutrino masses with $B-L$ gauge symmetry and $S_{3}$ flavor symmetry, Phys. Lett. B $\mathbf{7 4 1}$ (2015) 217.

[6] E. Ma, Naturally small seesaw neutrino mass with no new physics beyond the TeV scale, Phys. Rev. Lett. 86 (2001) 2502.

[7] E. Ma, Linkage of dirac neutrinos to dark U(1) gauge symmetry, Phys. Lett. B 817 (2021) 136290.

[8] E. Ma, Dirac neutrino mass matrix and its link to freeze-in dark matter, Phys. Lett. B $\mathbf{8 1 5}$ (2021) 136162.

[9] E. Ma, Dark matter from $\mathrm{SU}(6) \rightarrow \mathrm{SU}(5) \times \mathrm{U}(1)_{N}$, Phys. Rev. D 103 (2021) L051704. 
[10] H. Georgi and S.L. Glashow, Unity of all elementary-particle forces, Phys. Rev. Lett. 32 (1974) 438.

[11] K. Inoue, A. Kakuto and Y. Nakano, Unification of the lepton-quark world by the gauge group SU(6), Prog. Theor. Phys. 58 (1977) 630.

[12] S.K. Yun, Unified SU(6) gauge theory of the strong, weak, and electromagnetic interactions, Phys. Rev. D 18 (1978) 3472.

[13] Z. Chacko, P.B. Dev, R.N. Mohapatra and A. Thapa, Predictive dirac and majorana neutrino mass textures from SU(6) grand unified theories, Phys. Rev. D 102 (2020) . 